\title{
Reinvention of Depression Instruments by Primary Care Clinicians
}

Seong-Yi Baik, PhD ${ }^{1,2}$

Junius J. Gonzales, MD, MBA ${ }^{3}$

Barbara J. Bowers, $P b D^{4}$

Jean S. Anthony, $P b D^{5}$

Bas Tidjani, $P b D^{6,7}$

Jeffrey L. Susman, $M D^{2}$

'School of Nursing, University of

Louisville, Louisville, Kentucky

${ }^{2}$ Department of Family Medicine,

University of Cincinnati, Cincinnati, Ohio

${ }^{3}$ College of Behavioral and Community Sciences, University of South Florida,

Tampa, Florida

${ }^{4}$ School of Nursing, University of Wisconsin-Madison, Madison, Wisconsin

${ }^{5}$ University of Cincinnati College of Nursing, Cincinnati, Ohio

${ }^{6}$ School of Business, University of Dakar, Senegal, West Africa

${ }^{7}$ Department of African and African American Studies, University of Cincinnati, Cincinnati, Ohio

\begin{abstract}
PURPOSE Despite the sophisticated development of depression instruments during the past 4 decades, the critical topic of how primary care clinicians actually use those instruments in their day-to-day practice has not been investigated. We wanted to understand how primary care clinicians use depression instruments, for what purposes, and the conditions that influence their use.
\end{abstract}

METHODS Grounded theory method was used to guide data collection and analysis. We conducted 70 individual interviews and 3 focus groups $(n=24)$ with a purposeful sample of 70 primary care clinicians (family physicians, general internists, and nurse practitioners) from 52 offices. Investigators' field notes on office practice environments complemented individual interviews.

RESULTS The clinicians described occasional use of depression instruments but reported they did not routinely use them to aid depression diagnosis or management; the clinicians reportedly used them primarily to enhance patients' acceptance of the diagnosis when they anticipated or encountered resistance to the diagnosis. Three conditions promoted or reduced use of these instruments for different purposes: the extent of competing demands for the clinician's time, the lack of objective evidence of depression, and the clinician's familiarity with the patient. No differences among the 3 clinician groups were found for these 3 conditions.

CONCLUSIONS Depression instruments are reinvented by primary care clinicians in their real-world primary care practice. Although depression instruments were originally conceptualized for screening, diagnosing, or facilitating the management of depression, our study suggests that the real-world practice context influences their use to aid shared decision making - primarily to suggest, tell, or convince patients to accept the diagnosis of depression.

Ann Fam Med 2010;8:224-230. doi:10.1370/afm.1113.

\section{INTRODUCTION}

$\mathrm{D}$ epression is a highly prevalent, disabling, and costly public health condition in the United States, ${ }^{1-3}$ and more than one-half of people with mental health issues receive care from primary care clinicians. ${ }^{4}$ Despite decades of research, there remains a gap between ideal care for depression and actual outcomes in primary care settings. ${ }^{5,6}$ One strategy for enhancing depression management has been the development of depression instruments to help diagnose, stratify the severity of, and evaluate the treatment of depression. Since the development of the Hamilton Rating Scale for Depression, ${ }^{7}$ the Beck Depression Inventory, ${ }^{8}$ and the Zung Self-Rating Depression Scale ${ }^{9}$ in the early 1960 s, continued work in the development of depression instruments has led to the availability of numerous depression instruments today.

It is nearly impossible to identify all the depression instruments available today or to describe each instrument's purposes and performance. Salient dimensions of development efforts, however, have been the testingretesting of performance and comparing the instruments' psychometrics 
while attempting to better articulate their purposes, applicable settings, and target populations. ${ }^{10-17}$ With time, the trend has been toward the development and refinement of shorter instruments, such as the Primary Care Evaluation of Mental Disorders (PRIME-MD), Patient Health Questionnaire (PHQ-9), and PHQ$2,{ }^{10,15-20}$ as well as a change of focus from screening or case finding only to monitoring treatment and assessing management. ${ }^{15,20}$ More recently, some of these instruments also have been used in quality improvement interventions to diagnose, guide, and evaluate depression treatment outcomes. ${ }^{21,22}$

Despite the sophisticated development of depression instruments during the past 4 decades, how primary care clinicians use the instruments in their day-to-day practice settings remains unknown. This article reports on how and for what purposes primary care clinicians use depression instruments and the conditions that influence their use. This investigation was part of the Describing the Enigma of Evaluating Depression (DEED) project. Funded by the National Institutes of Health, this project investigates the care processes and real-world conditions for the recognition and management of depression and how depression instruments are used in that context. To our knowledge, the DEED project is the first to investigate how primary care clinicians use depression instruments in a real-world practice environment.

\section{METHODS}

\section{Theoretical Frame and Study Team}

Grounded theory method ${ }^{23,24}$ was used to understand primary care clinicians' reported use of and perspectives on depression instruments in their everyday practice. We used recruitment letters and consent forms, and we purposefully sampled on the basis of clinician type (general internist, family physician, and nurse practitioner), sex, years of practice, and type of practice environment. Our interdisciplinary research team comprised those from the disciplines of nursing, family medicine, anthropology, business, psychology, and psychiatry, who were of both sexes and of diverse ethnicity (white, West African, African American, and Asian). Our study was approved by the University of Cincinnati Institutional Review Board.

\section{Sampling and Sample}

Sampling was purposeful and iterative based on ongoing data analysis. For example, because earlier data analysis suggested a possible difference in the way patients convey depression and accept a depression diagnosis based on ethnicity and socioeconomic background, we subsequently sampled clinicians who serve high propor- tions of African American patients, indigent patients, and those who are less educated. Up to 3 clinicians from the same practice were allowed to participate in the study, which gave the research team the opportunity to take into consideration the potential influence of individual practice styles vs office practice environments. The final sample included 70 primary care clinicians (28 family physicians, 28 general internists, and 14 nurse practitioners) from 52 primary care offices of diverse financial structure and patient populations, including 7 solo practices and 4 federally qualified community health centers in the greater Cincinnati metropolitan area. The clinician sample included 24 men and 46 women (of the 46 women, 32 were physicians and 14 were nurse practitioners) of various ethnicity: 37 white (52.9\%), 23 African American (32.9\%), 9 Asian (12.9\%), and 1 Latino (1.4\%). The clinicians had between 1 and 30 years of practice experience. Eighteen of 52 offices served a mostly African American patient population.

\section{Data Collection}

Data were collected from 2005 to 2008 . The interviews were conducted by 3 of the study's investigators, all experienced with grounded theory interviews. Field notes documenting such contextual factors as the geographic location, office setting, and the interviews themselves were used as complementary data. Consistent with grounded theory method, the structure and the content of the interview questions evolved with time based on the analysis of ongoing data. Initial interview questions were semistructured but remained broad to allow participants to define the phenomenon from their perspective, describing what was relevant to the issue in their practice (Table 1). As the clinicians began to describe their experiences, we asked them to elaborate on topics most relevant to the study, emphasizing that "there are no right or wrong answers; we are here to understand primary care practice from your perspective." Thus, we allowed the data to emerge from the clinicians rather than imposing an initial theoretical construct. If clinicians specifically mentioned using or not using depression instruments, they were encouraged to discuss how and when they used them or how they made the choice not to use them. Care was taken to encourage reflective responses based on actual patients and ongoing practice rather than on an idealized or textbook world. We determined theoretical saturation of data ${ }^{23,24}$ when new conditions or influences regarding the use of depression instruments were not uncovered despite evaluating a diversity of clinicians and practice environments.

\section{Data Organization and Analysis}

All interviews were audio recorded and transcribed verbatim. NVivo 2 software (QSR International Pty 


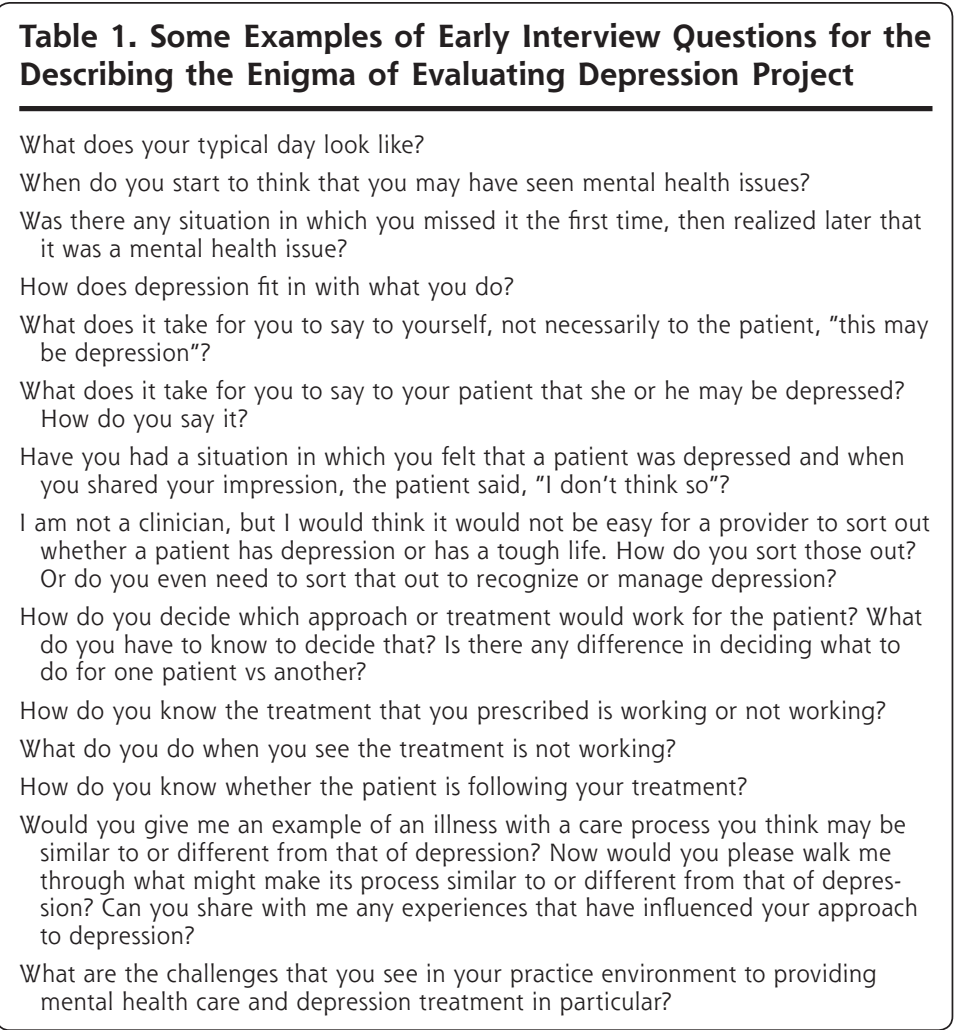

in the data collection or analysis. We then proceeded with a member check by presenting the preliminary data analysis to 3 focus groups of 24 clinicians who had previously participated in individual interviews in the study. The findings were corroborated and were refined based on this process.

\section{RESULTS}

The study found that clinicians reported they rarely used depression instruments for routine screening purposes or monitoring the treatment of depression, rather, they said they used the instruments to help foster patients' acceptance of their diagnosis. We identified 3 conditions that influenced the use of depression instruments: the extent of competing demands for the clinician's time, the lack of objective evidence, and the clinician's familiarity with the patient. Clinicians' assumption about patients' perception of the stigma of depression was a conceptual overarching influence on these 3 conditions.

Ltd, Victoria, Australia) and theoretical memos were used to organize the data analysis. The research team met weekly to discuss the data analysis, including ongoing sampling decisions and the evolution of interview questions. Grounded theory's constant comparative analysis method with open, axial, and selective coding guided the analysis. ${ }^{23,24}$ Open coding was used to identify the dimensions of using depression instruments (when, where, with whom, how, and for what purposes). Because our initial analysis indicated that clinicians used these instruments primarily to suggest, tell, or convince patients about their diagnosis, we focused on developing detailed descriptions of the conditions that influenced instrument use (axial coding). Selective coding was then applied to develop and refine the relationships between the conditions and the varied uses of instruments for diagnosing depression.

\section{Assuring Rigor}

Rigor and trustworthiness in our study were enhanced by comparing individual and group data analysis (line by line to paragraph by paragraph, depending on the stage of the analysis). We resolved differences in interpretation among team members by returning to the data to assure that interpretations remained grounded in participants' statements in context. In addition, we periodically discussed our analysis with a team of local and national consultants who were not involved
No differences were found in the reported use of depression instruments among the 3 clinician groups, nor did they prefer a specific instrument ${ }_{i}$ instead, they relied on what was available and quickly accessible in the office: "I still use the Beck's if I can't find the PHQ9 form or something" (family physician [FP] 11).

\section{Competing Demands}

Clinicians reported that competing demands for their time resulted in decreased use of depression instruments for screening or managing depression but increased their use as a way to suggest, tell, or convince the patient of their diagnosis. Clinicians described the challenge of sorting out a variety of diagnostic possibilities in a short time in patients with multiple problems.

I don't [use depression instruments]. I think a lot of it just comes down to the time factor. You know, we have instruments that the drug reps bring so much, lots of stuff in for us, for everything. Overactive bladder, migraine headaches, this and that...I personally don't use any of it (nurse practitioner [NP) 2).

Although experienced clinicians stated that the recognition of depression has become easier over time, finding an expedient way to tell and convince patients about the diagnosis in an environment of competing demands continued to pose a challenge. 
Quite honestly, those of us who are more experienced, you have a pretty good sense of who is depressed when they [patients] walk in and you start talking to them. But there, I'll be honest with you, I use the questionnaires more for people I feel who need that little bit of convincing (general internist [GI] 4).

\section{Lack of Objective Evidence}

The clinicians reported that depression instruments often helped make the symptoms of depression visible and more acceptable to the patient. Unlike physical illnesses, such as diabetes mellitus or hypertension, the absence of objective confirmatory tests (eg, a blood test) for depression that clinicians can show to a patient made it difficult for clinicians to present depression as a medical diagnosis. "I just can't draw your blood and say you're depressed, or do a CAT scan on your head" (FP 13). Clinicians therefore believed that the objectivity of a test (depression instrument) helped foster acceptance of depression, whether using these instruments in the office, directing patients to a Web site, or using the instrument as a homework assignment.

I think that little test they take like the Zung or the Beck, and they answer those, then I say, "Well, see, look, you know this indicates that you are depressed" (FP 1).

Thus, clinicians reported they used depression instruments to make depression visible and comparable to medical illnesses with objective criteria for diagnosis.

\section{Clinician's Familiarity With the Patient}

The clinician's familiarity with the patient streamlined the care for depression and obviated the need for the use of depression instruments. Familiarity helped clinicians to recognize symptoms of depression expeditiously, convey the diagnosis comfortably, and eliminate the need for unnecessary evaluation to rule out physical illnesses.

The duration and the nature of the patient-clinician relationship were 2 salient dimensions in developing familiarity, although there is considerable conceptual variation in how clinicians gain familiarity with each individual patient. As a result of increased familiarity, clinicians reported they could be more direct about the diagnosis of depression. According to the clinicians, familiarity helped them recognize a change in the patient and helped them convey this change in a nonjudgmental fashion: "I'll have someone who comes in, and I'll walk in the room and I say, 'Oh, gee, you don't seem to be your usual self'"' (GI 2). This approach was possible only when the clinician had a relationship with the patient. Knowing the patient also enabled the clinician not only to gauge how a patient might react to the diagnosis but also to take advantage of the patient's social support system for the purpose of enhancing treatment adherence.

What I see as the bigger side of it [knowing patients and their support system] is that after they've been [initially] treated, and the family notices the change in them, "this is how you used to be, and we didn't like that other person," then there's a lot of encouragement of "you need to stay on that medicine" (FP 9).

Clinicians addressed the necessity of being careful in presenting the diagnosis, especially with unfamiliar patients, because they have learned that more direct approaches often push patients away, resulting in the loss of opportunity to treat patients at all. They described experiences with unfamiliar patients who did not return after being given a diagnosis of depression:

They would perceive that as, "this guy, this doctor is judgmental and he doesn't even know me and he's already drawing these conclusions." He [the patient] will not like it he won't accept it if you say, "you're depressed," until you have some confidence and rapport with that patient... they [patients] might run away and go to a series of more doctors (GI 10).

As a result, clinicians described a lengthy process in presenting the diagnosis and agreeing on treatment for patients with whom they are unfamiliar:

Sometimes just plant that seed in their head, you may not fix them that time. If a patient comes in, and they have a lot of health complaints that you think could be attributable to their depression, and you can tell that they're not open to the diagnosis of depression, I try giving them a benefit of doubt, doing an evaluation, evaluating them for an underlying medical disease. And then seeing them back so that they get comfortable with you...sometimes I'll say, "We'll just kind of look at these things and we'll see what we find out here and, you know, see if you might have a family history of depression" (GI 3).

\section{Overarching Influence of Stigma}

Rather than a medical diagnosis, clinicians described patients viewing depression as a personal attribute or shortcoming that needed to be gingerly broached. Even clinicians who took pains to describe depression as being like any other medical illness thought that it was courteous to broach the subject indirectly, in a gentle manner, and that using a depression instrument enabled them to do that. Their use of an instrument was described as a strategy to "plant a seed in the patient's head" or "give the patient time to mull it over" - a method to gently convince patients that they may be depressed. 
Once you try to broach depression, then you really see, like in their body language, "No, that's not what it is, I am not depressed." They may not come out and say that, but you just know the way they're sitting [interviewee mimics the patient's sitting position, leaning back away from the doctor], the body language you know that they're not really in agreement. And so that would be if you have them [patients] to read about it [a depression instrument], to do PHQ-9 questionnaire, give them some time to mull it over, and then they may be more willing to accept it (FP 3).

Clinicians reported they used a depression instrument not necessarily to clarify the diagnosis but after they were sure the patient was depressed; they said they used it often in the process of negotiating with the patient to arrive at a shared meaning of illness and a treatment plan. The clinicians emphasized that patients had to accept the diagnosis before rendering treatment and that this acceptance is a major factor in the patient's adherence to the treatment. Thus, clinicians assumed patients viewed depression as a stigmatizing illness that had to be broached carefully, and that ongoing care depended upon a negotiated and shared treatment plan.

\section{DISCUSSION}

The invention of depression instruments was initially conceptualized to aid in the screening or case finding for depression or, more recently, to assist in quality-ofcare improvement interventions. The critical question of how these instruments are used in real-world primary care practices in the United States, however, has been left unanswered.

It is clear from this study that depression instruments are seldom used for broad-scale routine screening in the usual practice setting. In addition, such screening may only exacerbate the challenge of competing demands and may not assist in dealing with the real-world challenges that primary care clinicians face when they deliver depression care. The clinicians from the study clearly suggest that the dance (negotiating process) ${ }_{1}^{25}$ not the recognition of depression per $\mathrm{se}$, is a major challenge in providing depression care. Ironically, classical descriptions of the management of depression begin at the point of diagnosis, ${ }^{26}$ thereby not acknowledging the real-world challenge of the negotiation process between clinician and patient to reach a shared understanding and treatment agenda. Grounded theory, ${ }^{23,24}$ an interpretive research method specifically designed to illuminate human actions and conditions that influence actions, helped us to describe in detail how the clinicians' practice context and their interactions with patients influence their use of depression instruments-not the way they are initially conceptualized but to deal with a real-world practice challenge by facilitating an acceptance of the depression diagnosis and a shared treatment agenda.

A clinician's familiarity with the patient consistently emerged as an important condition for the recognition ${ }^{27}$ and management of depression, which can mitigate the challenges of competing demands, stigma, and the absence of objective evidence for diagnosing depression. A clinician's familiarity with the patient assists in recognizing changes in the patient that signify depression, obviates a lengthy negotiation process, breeds trust, and expedites a shared agenda. The analysis of our data suggests 2 salient dimensions of familiarity - the duration and the nature of the patient-clinician relationship. Continuity of care (seeing a patient over time) is a facilitator of a clinician's familiarity. There is, however, considerable conceptual variation in how clinicians gain familiarity with each individual patient. Further investigation into this conceptual inquiry would be beneficial to our ability to expedite the process.

The clinicians in this study, collectively, reflected a sociocultural perception of depression as a stigmatizing condition, a personal shortcoming rather than a medical illness. Even clinicians who believed that depression is a medical illness discussed the need to broach this diagnosis carefully with patients. This ambivalence appears to reflect societal beliefs surrounding depression and might inadvertently reinforce the patients' perception of stigma and divert a powerful opportunity to frame depression care in a positive and proactive manner. Further research should explore the influence of primary care clinicians' framing of depression on patients' attitudes and outcomes.

This study has several major limitations. It is based on clinicians' self-reports and may not accurately represent their actual practice. Clinicians' descriptions of care might have been idealized to reflect socially acceptable answers. Even so, clinicians were quite candid in giving detailed examples from their practice that one might consider socially undesirable, and the selfreport nature of the data did give insight into physicians' thought processes in using depression screening instruments. No independent measures of depression instrument use by either the clinicians or their staff were available. Nor could we fully assess the influence of differences in office environments (eg, patient populations served and systems of care) and larger contexts, such as urban vs rural environments. Finally, these results reflect findings from a Midwestern US sample and may not be generalizable to other populations.

The study findings raise critical conceptual and policy questions about how we can sensibly and sustainably diffuse an innovation and enhance its adaptation in a real-world primary care practice. Our study clarifies 
the advisability of requiring routine use of depression instruments in primary care settings in a world of competing demands and limited resources. ${ }^{28-30}$ The findings also challenge the assumption that the provision and the receipt of care ${ }^{31}$ are the same, as is conceptualized by most depression care guidelines. Whereas most guidelines begin with the diagnosis of depression and assume that the provision of treatment can begin immediately, our study suggests an often lengthy period of negotiation before receiving care. This conceptual gap between the provision of care and the receipt of care seems to align closely with the gap between idealized and real-world management of depression. This practice gap could have a major influence on care outcomes and, if not addressed properly, may lead to clinician frustration with current guidelines and reimbursement structures that overlook the contextual factors and situations which influence care receipt in real-world practice. Addressing this practice gap is urgent. The United States faces deficits of up to 44,000 primary care clinicians by the year 2025, which will result in about a $29 \%$ increased workload for family physicians and general internists for adult populations. ${ }^{32,33}$

To our knowledge, this study is the first to investigate the use of depression instruments based on clinicians' reports, and findings suggest a classic diffusion of technology with the repurposing of an innovation as it is diffused into the real world of primary care practice. Our findings are consistent with Roger's diffusion of innovation theory and the definition of reinvention. ${ }^{34}$ Rogers defines the concept of reinvention as "the degree to which an innovation is changed or modified by a user in the process of its adoption and implementation. ${ }^{34(\mathrm{p} 80)}$ Initially, depression instruments were viewed as an important means to correctly identify patients with depression and foster more timely treatment. The clinicians in our study, however, clearly indicated that routine screening and case finding do not fit in the real world of competing demands and limited resources in their primary care practice. Nor is the recognition of depression as such a real challenge. Accordingly, these instruments have been reinvented to deal with a real-world problem: convincing patients, believed to be depressed, that they are depressed and developing a shared agenda for treatment. Indeed, this initial dance is overlooked by current guidelines for depression, yet this challenge is deemed one of the greatest burdens imposed upon clinicians practicing in primary care. Future health services research and policy work in depression care warrant further investigation into ways to better foster familiarity, enhance the process of arriving at a shared agenda, and catalyze a more efficient process of care that bridges the gap between idealized and real-world practice.
To read or post commentaries in response to this article, see it online at http://www.annfammed.org/cgi/content/full/8/3/224.

Key words: Depression; primary health care, diffusion of innovation; quality of health care; physician's practice patterns

Submitted August 12, 2009; submitted, revised, October 22, 2009; accepted November 25, 2009.

Funding support: This study was supported by a grant from the National Institutes of Mental Health, NIH/NIMH R34 MH 071719.

Preliminary findings of this study were presented at AcademyHealth, June 4, 2007, Orlando, Florida; the 136th annual meeting and exposition of the American Public Health Association, October 27, 2008, San Diego, California; and 36th annual meeting of the North America Primary Care Research Group, November 18, 2008, Rio Grande, Puerto Rico.

\section{References}

1. Kessler RC, Demler O, Frank RG, et al. Prevalence and treatment of mental disorders, 1990 to 2003. N Engl J Med. 2005;352(24): 2515-2523.

2. Satcher D. Executive summary: a report of the Surgeon General on mental health. Public Health Rep. 2000;115(1):89-101.

3. Donohue JM, Pincus HA. Reducing the societal burden of depression: a review of economic costs, quality of care and effects of treatment. Pharmacoeconomics. 2007;25(1):7-24.

4. Regier D, Narrow W, Rae D, Manderscheid R, Locke B, Goodwin F. The de facto US mental and addictive disorders service system. Epidemiologic catchment area prospective 1-year prevalence rates of disorders and services. Arch Gen Psychiatry. 1993;50(2):85-94.

5. Katon W, von Korff M, Lin E, Bush T, Ormel J. Adequacy and duration of antidepressant treatment in primary care. Med Care. 1992;30(1):67-76.

6. Trivedi MH, Lin EH, Katon WJ. Consensus recommendations for improving adherence, self-management, and outcomes in patients with depression. CNS Spectr. 2007;12(8)(Suppl 13):1-27.

7. Hamilton M. A rating scale for depression. J Neurol Neurosurg Psychiatry. 1960;23:56-62.

8. Beck AT, Ward CH, Mendelson M, Mock J, Erbaugh J. An inventory for measuring depression. Arch Gen Psychiatry. 1961;4:53-63.

9. Zung WW. A self-rating depression scale. Arch Gen Psychiatry. 1965; 12:63-70.

10. Kroenke K, Spitzer RL, Williams JBW. The Patient Health Questionnaire-2: validity of a two-item depression screener. Med Care. 2003; 41(11):1284-1292.

11. Henkel V, Mergl R, Kohnen R, Allgaier A-K, Möller H-J, Hegerl U. Use of brief depression screening tools in primary care: consideration of heterogeneity in performance in different patient groups. Gen Hosp Psychiatry. 2004;26(3):190-198.

12. Shafer $A B$. Meta-analysis of the factor structures of four depression questionnaires: Beck, CES-D, Hamilton, and Zung. J Clin Psychol. 2006;62(1):123-146.

13. United States Preventive Services Task Force. Guide to Clinical Preventative Services. Periodic Updates: Screening For Depression, Agency For Healthcare Research and Quality. 3rd ed. Rockville, MD: US Department of Health and Human Services; 2002.

14. Gilbody S, Richards D, Brealey S, Hewitt C. Screening for depression in medical settings with the Patient Health Questionnaire (PHQ): a diagnostic meta-analysis. J Gen Intern Med. 2007;22(11):1596-1602.

15. Kroenke K, Spitzer RL, Williams JBW. The PHQ-9: validity of a brief depression severity measure. J Gen Intern Med. 2001;16(9):606-613. 
16. Spitzer RL, Kroenke K. Williams JB. Validation and utility of a selfreport version of PRIME-MD: the PHQ primary care study. Primary care evaluation of mental disorders, patient health questionnaire. JAMA. 1999;283(18):1737-1744.

17. Spitzer RL, Williams JBW, Kroenke K, Hornyak R, McMurray J. Validity and utility of the PRIME-MD patient health questionnaire in assessment of 3000 obstetric-gynecologic patients: the PRIME-MD Patient Health Questionnaire Obstetrics-Gynecology Study. Am J Obstet Gynecol. 2000;183(3):759-769.

18. Volk RJ, Pace TM, Parchman ML. Screening for depression in primary care patients: Dimensionality of the short form of the Beck Depression Inventory. Psychol Assess. 1993;5(2):173-181.

19. Rouch-Leroyer I, Sourgen C, Barberger-Gateau P, Fuhrer R, Dartigues JF. Detection of depressive symptomatology in elderly people: a short version of the CES-D scale. [Milano]. Aging (Milano). 2000;12(3):228-233.

20. Löwe B, Kroenke K, Gräfe K. Detecting and monitoring depression with a two-item questionnaire (PHQ-2). J Psychosom Res. 2005; 58(2):163-171.

21. Lowe B, Unutzer J, Callahan CM, Perkins AJ, Kroenke K. Monitoring depression treatment outcomes with the Patient Health Questionnaire-9. Med Care. 2004;42(12):1194-1201.

22. Gaynes BN, Rush AJ, Trivedi MH, et al. Primary versus specialty care outcomes for depressed outpatients managed with measurement-based care: results from STAR*D. J Gen Intern Med. 2008; 23(5):551-560.

23. Strauss AL. Qualitative Analysis for Social Scientists. Cambridge: Cambridge University Press; 1987.

24. Bowers BJ. Grounded theory. In: Sarter B, ed. Paths to Knowledge: Innovative Research Methods for Nursing. Washington DC: National League for Nursing; 1989:33-59.
25. Miller WL, McDaniel RR Jr, Crabtree BF, Stange KC. Practice jazz: understanding variation in family practices using complexity science. J Fam Pract. 2001;50(10):872-878.

26. Agency for Health Care Policy and Research. Depression in Primary Care: Detection and Diagnosis. Clinical Practice Guideline Number 5. Rockville, MD: US Department of Health and Human Services; 1993.

27. Baik SY, Bowers BJ, Oakley LD, Susman JL. The recognition of depression: the primary care clinician's perspective. Ann Fam Med. 2005;3(1):31-37.

28. McAlpine DD, Wilson AR. Screening for depression in primary care: what do we still need to know? Depress Anxiety. 2004;19(3):137-145.

29. Palmer SC, Coyne JC. Screening for depression in medical care: pitfalls, alternatives, and revised priorities. J Psychosom Res. 2003;54(4):279-287.

30. Gilbody S, House AO, Sheldon TA. Screening and case finding instruments for depression. CMAJ. 2005;(4):CD002792.

31. Starfield B. Primary Care; Balancing Health Needs, Services, and Technology. New York, NY: Oxford University Press; 1998:438.

32. Colwill JM, Cultice JM, Kruse RL. Will generalist physician supply meet demands of an increasing and aging population? Health Affairs 2008; April 29:w232-40.

33. Kuehn BM. Reports warn of primary care shortages. JAMA. 2008; 300(16):1872-1875, 1874-1875

34. Rogers EM. Diffusion of Innovation. 5th ed. New York, NY: Free Press: 2003:551. 